\title{
Plus Ou Moins Que Deux: Le Tašdīd Dans La Tradition Métrique Persane
}

\section{Citation}

Landau, Justine. 2018. Plus Ou Moins Que Deux: Le Tašdīd Dans La Tradition Métrique Persane. Bulletin D'études Orientales 65, no. 1: 135-156.

\section{Permanent link}

http://nrs.harvard.edu/urn-3:HUL.InstRepos:41542963

\section{Terms of Use}

This article was downloaded from Harvard University's DASH repository, and is made available under the terms and conditions applicable to Other Posted Material, as set forth at http:// nrs.harvard.edu/urn-3:HUL.InstRepos:dash.current.terms-of-use\#LAA

\section{Share Your Story}

The Harvard community has made this article openly available.

Please share how this access benefits you. Submit a story.

\section{Accessibility}




\title{
Plus ou moins que deux : le tašdìd dans la tradition métrique persane
}

\author{
Justine LANDAU
}

Résumé : Tašdid : « tekrār-e harf », « [signe de] renforcement ». En persan, comme en arabe, toute lettre portant la šadda compte pour deux. Attribué à al-Hुalïl b. Ahmad (ob.ca. 796), le signe suscrit en forme de sin était d'abord destiné à marquer les consonnes géminées (mošaddad) dans les poèmes consignés par écrit. Avant de s'imposer comme diacritique incontournable dans les textes classiques et modernes, l'inscription du tašdìd procédait en effet d'un souci d'exactitude métrique. En arabe, où la gémination a valeur distinctive, ce motif initial fut rapidement oublié. C'est toutefois en persan, non sans quelque ironie, que la vertu métrique de la šadda devait être ranimée. Or, en rendant sa raison d'être à cette petite notation, la tradition métrique persane en déplaçait aussi la cible. En persan, le tašdìd participait désormais d'une stratégie d'adaptation de la métrique halîlienne ('arūẹ) dans une langue insensible au redoublement consonantique. Le présent article examine cette facette intrigante de la gémination persane, peu étudiée à ce jour.

Mots-clés : tašdīd, gémination, métrique persane, Šams-e Qeys-e Rāzī, Nașīr al-Dīn Ṭūsī.

\begin{abstract}
Tašdìd: "tekrār-e harf" "[sign of] emphasis." In Persian, like in Arabic, any letter bearing the šadda should be read as two. Attributed to al-Halī b. Ahmad (ob.ca. 796), the sin-shaped superscript was first designed to mark geminated (mošaddad) consonants in poetry set down in writing. Before becoming the widespread diacritic that we know today, the šadda thus originated from a metrical concern. In Arabic, where gemination is distinctive, this primal focus may have been forgotten. Somewhat ironically, the prosodic purpose of the šadda was later revived in Persian poetry. While recovering its initial design, however, the Persian metrical tradition also slightly altered its target. In Persian, the tašdìd became a device to implement Halilian metrics ('arū $\bar{d}$ ) in a language insensitive to consonant length. It is the object of our paper to examine this intriguing facet of Persian gemination, which remains widely unexplored.
\end{abstract}

Keywords: tašdìd, gemination, Persian metrics, Šams-e Qeys-e Rāzī, Nașīr al-Dīn Ṭūsī. 


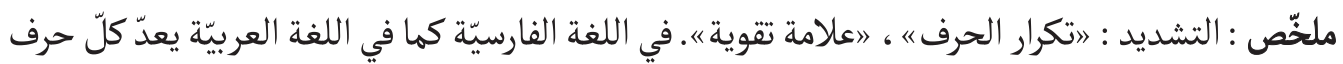

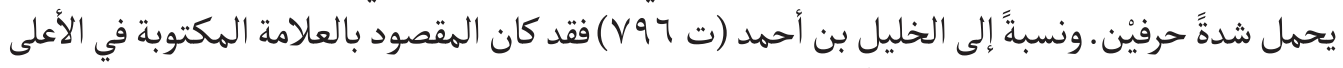

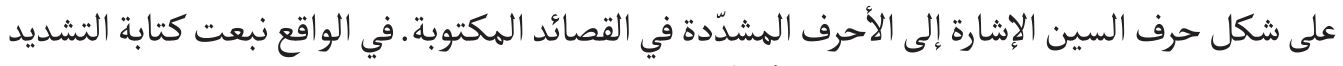

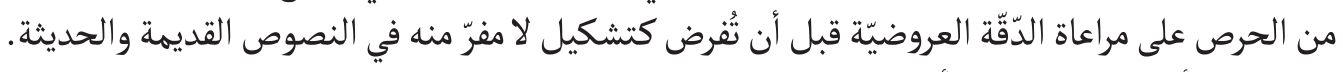

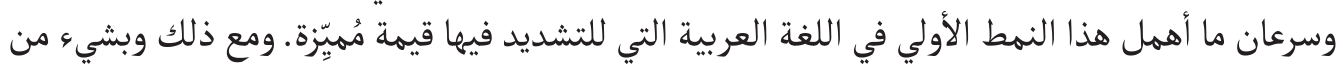

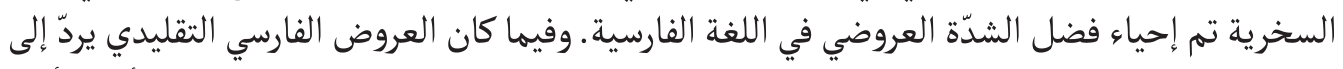

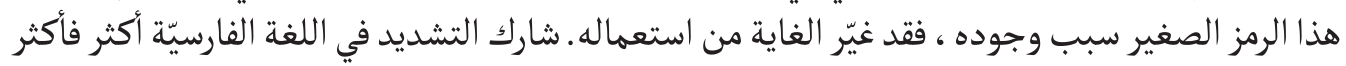

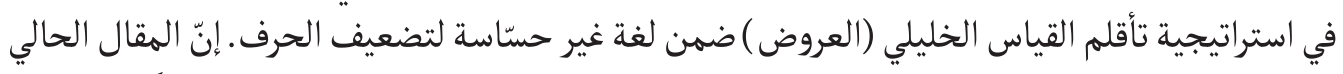

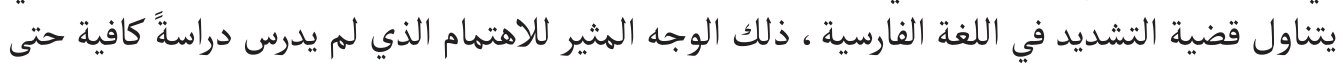

الكلمات الهحوريّة : تشديد ، تضعيف ، القياس الفارسيّ ، شمس القيس الرّازي ، نصير الدين طوسي. 


\section{Introduction 1}

Ce fut d'abord un petit point vert - ou bleu, parfois rouge - tracé au-dessus d'une consonne, aux côtés d'autres points de couleur représentant qui une voyelle, qui un tanwīn, ou encore une hamza, dans les plus anciens manuscrits du Coran ${ }^{2}$. La šadda, marque du redoublement de consonne en arabe, n'en est venue que progressivement à se distinguer dans l'écriture. De fait, s'ils participent de la splendeur des manuscrits désignés sous le nom de « kufiques », les diacritiques aux pigments éclatants des premiers siècles de l'Islam étaient encore fort rudimentaires. Dans certains manuscrits, un même point bleu pouvait servir à indiquer une voyelle brève ou un tašdìd; dans d'autres, le rouge était réservé aux voyelles, le vert à la hamza et le jaune à la šadda ; ailleurs encore, on trouve de petits cercles rouges, voire de simples traits, en lieu et place des points. Loin d'être fixé, et encore moins unifié, le code des couleurs était susceptible de varier d'une région ou d'un copiste à l'autre ${ }^{3}$, si bien que certaines notations font, aujourd'hui encore, l'objet d'interprétations divergentes parmi les spécialistes ${ }^{4}$.

C'est à al-Hुalì b. Ahmad (ob.ca.796) que la tradition attribue le perfectionnement des diacritiques dans l'écriture arabe. En particulier, c'est à al-Halīl que l'on devrait d'avoir remplacé le point par le sin suscrit caractéristique ( $\left.\_\right)$, abréviation de šadíd (redoublé, renforcé) qui s'est imposée comme le signalement des consonnes géminées 5 . Dans The Arabic Language, Versteegh précise que cette réforme se serait développée dans la transcription des textes poétiques avant d'être étendue aux manuscrits coraniques ${ }^{6}$. Certes, on sait les précautions qu'impose l'attribution à un seul individu d'une réforme de si grande portée. Il y a fort à parier que l'enchaînement réel des initiatives, singulières ou collectives, qui ont abouti au renouvellement de l'écriture arabe resteront longtemps insondables. Pourtant, en assignant les nouvelles conventions diacritiques au célèbre « inventeur » de la métrique arabe, la tradition savante affirmait tout de même quelque chose. Elle entérinait l'existence d'un rapport essentiel entre la désambiguïsation des signes graphiques et la lecture correcte de la poésie.

Une chose est sûre : le succès du petit sinn ne s'est pas démenti. Traversant les frontières avec l'extension de l'islam, il sut se rendre aussi indispensable que l'alphabet arabe parmi les langues de la umma. On le retrouve notamment dans les plus anciens manuscrits de l'Iran musulman, région qu'il n'a jamais quittée depuis. D'un point de vue linguistique, cependant, le phénomène désigné par l'exposant à deux dents n'est pas absolument identique en arabe

1. Cet article reprend un point soulevé dans ma thèse de doctorat, pour le développer dans une autre perspective, à la lumière de récents travaux sur la gémination en persan. Voir LANDAU 2013, p. 107-110.

2. Voir DUTTON 1999.

3. Ibid. Voir aussi SOURDEL-THOMINE 1978, p. 1119-1120.

4. Voir par exemple RIEU 1894 et DÉROCHE 1992.

5. VersteEgh 1997, p. 55-57. Parmi les huit ouvrages qui lui sont attribués, le Fihrist recense un Kitāb al-nuqt wa-l-šakl, traité sur les points diacritiques. Voir Ibn al-Nadīm, al-Fihrist, p. 43.

6. VeRSTEEGH 1997, p. 56-57. 
et en persan. Les phonéticiens reconnaissent ainsi qu'en persan, la gémination, appelée tašdìd (renforcement, insistance), n'a pas valeur d'opposition distinctive. Le placement convenable des géminées reste un indice de la compétence linguistique du locuteur, mais c'est en poésie que leur présence, ou leur absence, fait toute la différence. Parce qu'en poésie, une lettre de trop, ou de moins, défigure le vers et produit un mètre faux.

Les pages qui suivent n'ont pas vocation à produire un aperçu global de la gémination en persan, ni du diacritique qui lui correspond. Les réflexions présentées dans cet article ont seulement pour objet de mettre au jour un usage singulier, et proprement métrique du tašdìd, identifié par les théoriciens classiques de la poésie en persan. Pour y parvenir, il fallait néanmoins faire le point sur les usages persans de la gémination reconnus par les linguistes. Nous prenons appui pour ce faire sur deux synthèses récentes - souvent complémentaires, quelquefois discordantes-, fournies par Taqī Vahịidiyān Kāmyār (1371/1992) et 'Alī-Ašraf Șādeqī (1392/2013). Nous en venons ensuite aux questions spécifiques soulevées par le traitement des géminées dans la poésie persane. Enfin, nous présentons l'étrange cas du «tašdìd métrique », cet ancien allié des métriciens classiques souvent incompris des modernes.

\section{Tašdìd, vos papiers!}

La gémination, ou redoublement de consonne, est un phénomène phonétique circonscrit dans la chaîne parlée. Comme l'explique Troubetzkoy, « il y a dans beaucoup de langues des consonnes dites "géminées" ». Selon le père de la phonologie moderne, ces dernières

se distinguent des consonnes non géminées par leur durée plus longue et la plupart du temps aussi par une articulation plus énergique [...]. Mais en position intervocalique les consonnes géminées sont réparties entre deux syllabes, leur implosion appartenant à la syllabe précédente et leur explosion à la syllabe suivante. En outre ces consonnes géminées n'apparaissent que dans les positions où la langue en question admet des groupes de consonnes; elles exercent sur les sons environnants la même action que les groupes de consonnes et sont traitées en général exactement comme les groupes de consonnes (TROUBETZKoY 1949, p. 184). ${ }^{7}$

Bien que fort courant, le phénomène a des implications variées. Dans certaines langues, la gémination détermine des oppositions phonologiques distinctives ${ }^{8}$, comme c'est le cas en arabe, en japonais ou en italien. Dans la majorité des langues du monde, en revanche, il n'en est rien; il en va ainsi du persan, du français, ou encore de l'anglais, considérés comme insensibles à la longueur consonantique. Ces différences nous alertent sur le fait que la notation écrite n'est pas le tout du phénomène. Si l'on songe à la šadda en particulier, que désigne-t-elle au juste dans les langues qui, à la suite de l'arabe, l'ont adoptée comme

7. Voir aussi TROUBETZKoY 1949, p. 184-189; p. 201 sq. ; p. 215.

8. TROUBETZKoy 1949 , p. 33-46. 
marque de la gémination? Est-elle partout l'indice d'une opération identique ? Plusieurs séries d'exemples semblent suggérer que non.

Sur le domaine persan, peu de travaux sont consacrés en propre à la gémination. Dans les vingt dernières années, deux linguistes iraniens ont toutefois livré sur le sujet des contributions importantes. Il s'agit d'une part du phonéticien T. Vahịidiyān Kāmyār qui, dans un article aussi bref que dense (VAḤīDIYĀN KĀMYĀR 1992), s'attache à exposer les convergences entre la description linguistique de la gémination et sa représentation traditionnelle en persan, résolvant au passage une difficulté liée au tašdìd final de mot. Plus récemment, A.-A. Șādeqī (2013) a proposé une véritable synthèse sur la gémination en persan, avec esquisse d'évolutions diachroniques et typologie des usages recensés. Le descriptif qui suit reprend largement les données fournies par ces auteurs, et une partie de leurs analyses.

Le premier souci des auteurs est d'interroger la nature des géminées en persan. Ils identifient en réalité non pas un, mais deux types de tašdìd en usage dans la langue. Ils distinguent d'une part un tašdìd "de souche », et d'autre part un tašdìd "d'adoption ", entré en persan avec les mots d'emprunt - qui furent massifs dans les premiers siècles de l'Islam, et très majoritairement arabes. S'il importe aux linguistes de traiter séparément ces deux types de tašdìd, c'est que la gémination, on l'a vu, a un statut très différent dans ces deux langues : distinctive, elle joue en arabe un rôle crucial dans l'organisation du lexique ; en persan, en revanche, elle n'a pas ce caractère discriminant. À cette exception près qu'en persan courant, la proportion élevée de mots arabes suggère l'existence d'un conflit des oppositions phonétiques distinctives, et de leur perceptibilité par les locuteurs.

Il semblerait aussi que nos auteurs ont été marqués par une lecture commune: le traité de prosodie du célèbre philosophe et savant Nașīr al-Dīn Țūsī (1201-1274), Mecyār al-aš'är, dar 'elm-e 'arud va qavāfí (L'étalon des poésies, de la science de la métrique et des rimes). Daté de 649/1251, ce texte compte parmi les plus anciens témoins de la pensée classique de la langue persane. Une phrase en particulier a retenu l'attention des linguistes. Dans un chapitre consacré à « la qualité relative des lettres vocalisées et quiescentes en poésie », Nașiir al-Dīn écrit: «Dans l'ensemble, mieux vaut n'employer le tašdìd que le moins possible dans la langue persane, car il n'y est pas originel ${ }^{9}$ ». Vahịdiyān Kāmyār et Șādeqī se penchent l'un et l'autre sur le sens de cette affirmation. Le philosophe estimait-il la gémination inconnue du persan hors vocabulaire arabe ? L'examen diachronique de la langue s'oppose à cette interprétation. Les linguistes penchent plutôt pour une hypothèse sémantique et contextuelle. Pourtant, une lecture attentive de leurs analyses fait apparaître un doute, subtil mais persistant : se pourrait-il que le tašdìd ne soit qu'un intrus en persan?

9. TṬūsī, Me yār al-ašcār, p. 179 : «va bar jomle, čandān ke dar log்at-e pārsī tašdīd kamtar āvarand behtar bāšad, če tašdīd dar ān log̉at aṣli nīst ». Sauf indication contraire, toutes les traductions du persan sont de l'auteur. 


\section{Persan « d'adoption»}

En arabe, la gémination a valeur distinctive : elle est essentielle au sens des mots et permet de distinguer des paires. Le tašdìd fait partie intégrante de certains schèmes verbaux et nominaux, comme les formes verbales II ( $\left.f a^{\prime \prime} a l a\right)$, V (tafa" ${ }^{\prime \prime} a$ la) et IX (if'alla). Dans certains cas, le redoublement consonantique porte une valeur sémantique d'intensif, par opposition au verbe simple. C'est ce qui permet de distinguer kasara (il a cassé ; coupé en morceaux) de kassara (il a coupé en petits morceaux) ; ou encore šabata (il a frappé) de šabbata (il a beaucoup frappé) (ȘĀDEQĪ 1392/2013, p. 3).

Pour l'arabe, W. Wright (1951, p. 13-16) recense en réalité un tašdìd « nécessaire » et un tašdìd « euphonique ${ }^{10}$ ». Le tašdìd " nécessaire » est celui qui « indique un redoublement dont dépend la signification du mot ». Il permet de distinguer des paires comme amara (il a commandé) et 'ammara (il a nommé un commandant), ou murr (amer) et *mur (inexistant dans la langue). Dans la chaîne phonique, ce tašdìd est toujours précédé d'une voyelle. À l'inverse, le tašdìd « euphonique » se rencontre à la suite d'une consonne muette qui n'est pas prononcée mais assimilée à la consonne suivante : c'est la géminée que l'on trouve notamment à la jonction avec l'article al-, lorsqu'il est suivi de l'une des lettres dites solaires, comme dans ar-raḥmān ou aš-šams ${ }^{11}$, ainsi que dans d'autres cas d'assimilation.

Qu'en est-il des mots d'emprunt en persan? Les mots d'origine arabe sont nombreux dans le lexique, et ce, depuis l'islamisation du plateau iranien au VII ${ }^{\mathrm{e}}$ siècle de l'ère commune. La question qui se pose est alors la suivante : en accueillant un nombre élevé de vocables arabes, les persanophones ont-ils parallèlement développé une sensibilité à la gémination distinctive ? Sur ce point, les avis divergent. Vahīdiyān Kāmyār (1371/1992, p. 27) tient que l'entrée massive de mots arabes s'est accompagnée en persan d'une sensibilisation à la valeur d'opposition distinctive du tašdìd dans les mots d'emprunt. C'est ainsi, explique-t-il, que les persanophones font la différence entre ban $\bar{a}^{2}$ (construction, édifice) et bann $\bar{a}^{2}$ (maçon). Sans renier l'existence de telles paires, Șādeqī (1392/2013, p. 37) insiste sur le fait que les mots d'emprunt sont majoritairement traités dans la langue comme des mots persans ${ }^{12}$. La preuve en est que les géminées sont sujettes en persan à une évolution phonologique identique, quelle que soit leur origine. Ainsi la tendance à la simplification des géminées touche aussi bien les mots d'origine arabes (ȘĀDEQī 1392/2013, p. 33-35) : ammā > amā (mais) ; ballūt > balūt (gland) ; tannūr > tanūr (four) ; hammām > hamūm (bain) ;

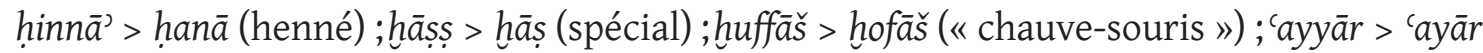
(aloi) ${ }^{13}$. Pour le linguiste, l'argument du traitement uniformisé des vocables dans la langue

10. Pour une description plus complète, on se reportera à FLEISCH 1961, en part. § 4 et $\S 50 h$; et FLEISCH 1971. Voir aussi ALFOZAN 1989. Pour un aperçu des descriptions fournies dans la tradition linguistique arabe, voir BOHAS, Guillaume et Kouloughli 1990, « Gemination (idjām) », p. 90-91.

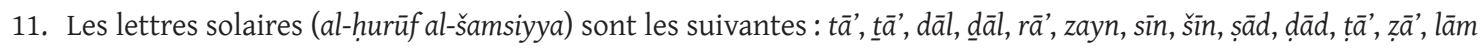
et nūn.

12. Voir aussi ȘĀDEQİ 1975.

13. Ce que VAḤ̄DIYĀN KĀMYĀR 1371/1992, p. 27 accorde volontiers. 
discrédite la thèse de l'importation de la gémination distinctive. De fait, en dépit d'une disproportion numérique largement favorable au tašdìd arabe, ce dernier n'est pas un cas isolé dans la langue persane.

Persan « de souche»

En persan, la gémination est un phénomène rare, mais pas inconnu. Seulement, son fonctionnement n'est pas exactement superposable à celui de l'arabe. Parmi ses usages "autochtones", en réalité, il convient de distinguer un tašdìd lexical et un tašdìd contextuel.

Tašdìd lexical

Le tašdid « lexical » est un redoublement de consonne qui se produit dans le radical d'un mot. À ce stade, ne sont envisagées que les géminations manifestées dans des vocables supposés "persans purs » (färsi-e sare) ${ }^{14}$. C'est le cas, par exemple, des mots arre (scie), barre (agneau), pelle (marche, escalier), tekke (pièces, morceau), čelle (période de quarante jours), horram (plaisant, joyeux), farroh (heureux, fortuné), kalle (tête) ou mazze (goût, saveur). On trouve chez A.-A. Șādeqī des développements consacrés aux évolutions historiques qui ont présidé à l'apparition de ces géminées. Le cas de čelle est exemplaire. À partir d'un mot čehele (de čehel, quarante), le redoublement consonantique résulte de deux transformations successives. Dans un premier temps, la chute du [h] provoque l'allongement compensatoire de la voyelle [e] subséquente : čehele > čeele > čèle. Dans un second temps, cette voyelle [ē] s'abrège en [e], ce qui a pour effet d'entraîner la gémination du [1] qui suit: čèle > *čele > čelle (ȘĀDEQī 1392/2013, p. 7). Plusieurs occurrences de tašdìd lexical s'expliquent de cette manière. D'autres résultent d'un phénomène de sandhi, dans les composés comme šab-bū (qui répand son parfum la nuit) > šabbü (giroflée) ou dans les dérivés comme dovvom/doyyom (deuxième $<$ do, deux) et sevvom/seyyom (troisième $<$ se, trois). Considérées en diachronie, cependant, la plupart des géminées persanes sont la conséquence d'une ancienne assimilation (edġām) (ȘĀDEQī 1392/2013, p.19-22), comme c'est le cas de pašše (moustique), du moyen-perse pahšag (réduction du groupe consonantique ȟš > šš) (ȘĀDEQİ 1392/2013, p. 19).

Pour preuve que la gémination n'est pas tout entier un phénomène d'emprunt, les auteurs rappellent qu'en outre, les consonnes redoublées se rencontraient déjà en moyen-perse. L'ancêtre historique du persan possédait par exemple les mots vattar (mieux), farrah (faste, splendeur) ou hwwarrah (gloire, aura) (VAḤīDIYĀN KĀMYĀR 1371/1992, p. 26). En revanche, on remarque que toutes les consonnes ne sont pas égales face à la gémination. En persan, les lettres les plus communément géminées sont le $[\mathrm{r}]$ «roulé »

14. L'expression, qui se retrouve à plusieurs reprises sous la plume de T. Vahīdiyān Kāmyār (1371/1992) et de A.-A. Șādeqī (1392/2013), a une histoire. Elle connotait d'abord, dans le premier tiers du Xx ${ }^{\mathrm{e}}$ siècle, les préoccupations d'un petit groupe de réformateurs qui proposait d'expurger la langue persane des mots d'origine étrangère, particulièrement arabes. Ces aspirations trouvèrent leur expression institutionnelle dans la fondation, en 1935, de l'Académie de Langue et de Littérature Persanes (Farhangestān-e zabān va adab-e fārsī), qui se prononce sur les usages de la langue persane et produit notamment un dictionnaire. A.-A. Șādeqī est membre de cette académie. 
et le [1] latéral, que leur instabilité articulatoire prédispose au redoublement, surtout en position intervocalique (ȘĀDEQİ 1392/2013, p. 26-29 ; VAḤīDIYĀN KĀMYĀR 1371/1992, p. 26). Les consonnes dites « liquides" (ravān) sont ainsi, plus que les autres, «sujettes à tašdìd» (tašdìd-padìr), selon la formule d'Adīb-e Solțānī (1354/1975) ${ }^{15}$. Cela, les anciens l'avaient bien perçu. Contemporain de Nașīr al-Dīn, Šams-e Qeys-e Rāzī (circ. 1175-1240) est l'auteur du manuel de référence classique de la poétologie persane, le Kitāb al-mu'jam fì ma'āyīr ašcār al-'ajam (Le livre de la somme sur les étalons des poésies des Persans). Dans son ouvrage, il signale « le cas de la consonne $r \bar{a}$ ' qui est répétée dans la prononciation et dont, pour cette raison, on dirait qu'il s'y trouve deux consonnes ». Šams-e Qeys en déduit ainsi que « le redoublement (tašdìd), appliqué à la consonne rā̉), n'est pas laid » (Šams-e Qeys, Kitāb al-mújam, p. 267 ; DIEBLER 1997, p. 211).

Ainsi donc, le persan connaît bien le redoublement de consonnes. Toutefois, la gémination étant dépourvue dans cette langue de fonction distinctive ${ }^{16}$, A.-A. Șādeqī considère comme fortuite la rencontre de doublets qui ne se différencient que par une consonne géminée, comme par exemple kore (sphère, globe), qui apparaît notamment dans l'expression kore-ye zamin (terre, globe terrestre), vs. korre (poulain, ânon). Ils constituent selon lui un cas d'homonymie accidentelle ${ }^{17}$. C'est le même argument qui permet d'expliquer la tendance générale du persan à la simplification des géminées: on parle alors d'allègement (tahfiff) ${ }^{18}$. Aux yeux de l'observateur, cette évolution a une conséquence remarquable : en persan moderne, nombre de vocables admettent les deux prononciations possibles ${ }^{19}$. Dans la langue familière ${ }^{20}$, on a ainsi indifféremment pašše ou paše (moustique), bačče ou bače (enfant), galle ou gale (troupeau), lappe ou lape (pois cassé), ommìd ou omid (espoir), tarre ou tare (poireau), šekkar ou šekar (sucre), avec une prédilection pour la forme allégée ${ }^{21}$. À l'inverse, les linguistes reconnaissent un redoublement consonantique propre à l'oral en la figure du tašdìd « d'insistance » ${ }^{22}$ ou " gémination expressive » ${ }^{23}, \mathrm{du}$ type âf(f)arin (bravo), ham(m)ǐše (toujours), où la consonne redoublée a valeur d'intensif.

Sous la rubrique des géminations propres au persan familier, il faut encore citer une catégorie remarquable :la gémination « analogique ${ }^{24}$.Ces redoublements consonantiques

15. Cité dans ṢĀDEQĪ 1392/2013, p. 26.

16. « Naqš-e tamāyoz-dahande-ye ma‘ānī ». Voir VAḤĪDIYĀN KĀMYĀR 1371/1992, p. 26 ; ȘĀDEQİ 1392/2013, p. 33.

17. ȘĀDEQĪ 1392/2013, p. 33.

18. Ibid.

19. ȘĀDEQĪ 1392/2013, p. 33-36 ; VAḤ̄DIYĀN KĀMYĀR 1371/1992, p. 26.

20. LAZARD 1957, p. 24.

21. Șādeqī fait cependant remarquer que certains mots résistent à la simplification des géminées et continuent d'être employés avec tašdīd, comme arre, pelle, tekke, horram, farroh, kalle, karre, gabbe, et d'autres, certains d'origine turque, ainsi tappe (« colline, coteau ») et jeǵge (« aigrette »). Voir ȘĀDEQİ 1392/2013, p. 37.

22. « Tašdīd-hā-ye 'āțefī va elqā'̄i » chez ȘĀDEQİ 1392/2013, p. 29-30. VAḤīDIYĀN KĀMYĀR 1371/1992, p. 27 parle de « fonction d'insistance »: " naqš-e ta'kīdī ».

23. LAZARD 1957, p. 24.

24. « Tašdīd-hā-ye qiyāsī ». ṢĀDEQĪ 1392/2013, p. 22-26. 
apparaissent en persan dans des mots d'emprunt qui, en arabe, en étaient dépourvus. Le redoublement s'explique alors par la ressemblance qu'entretiennent ces mots avec d'autres, dotés de géminées. Il s'agit d'une tendance générale à l'alignement sur un paradigme connu. On trouve ainsi ' $\bar{a} d \bar{l}$ (habituel, ordinaire < ' $\bar{a} d a t$, habitude, coutume) prononcé 'a $\bar{a} d \bar{l}$, peut-être sous l'influence de māddì (matériel). Or, le tašdìd "analogique », qui compte surtout des spécimens d'apparition récente, était déjà pratiqué par les poètes classiques. Ainsi Mowlānā Jalāl al-Dīn Rūmī (1207-1273) emploie le tašdìd dans des mots arabes où il n'a rien à faire, comme $f \bar{a}$ 'ed(d)e (profit, intérêt, avantage) ou qaț(t)ār (file), possiblement sur le modèle de 'atțār (parfumeur) ou de bazzāz (marchand d'étoffe, drapier) ${ }^{25}$.

Tašdìd final, tašdìd contextuel

Au XIII ${ }^{\mathrm{e}}$ siècle, Nașīr al-Dīn Ṭūsī reconnaissait déjà l'existence d'un tašdìd lexical. Il en donnait même quelques exemples : arre (scie), ġorrande (rugissant) et borrān (tranchant) ${ }^{26}$. Il s'agit, dans les deux derniers cas, d'une forme verbale nominalisée : un nom d'agent en -ande, et un gérondif présent en -ān. Ce type de tašdìd a ceci de particulier qu'il concerne la dernière consonne du radical verbal : gंorr- et borr-. Plus généralement, ces occurrences s'inscrivent dans la catégorie du tašdìd final de morphème ou de mot, que les linguistes traitent le plus souvent à part ${ }^{27}$. La raison en est simple : ces géminées ne se réalisent que dans certaines circonstances phonologiques précises ${ }^{28}$.

Sur cette question, T. Vahīiliyān Kāmyār est le plus explicite. À strictement parler, affirme le phonéticien, le tašdìd ne peut pas apparaître en finale absolue ${ }^{29}$. La règle est générale, puisqu'elle découle de la définition même de la gémination. Elle vaut donc indifféremment pour les mots arabes et persans. Selon la définition phonologique de base, en effet, une consonne est dite géminée si elle est traversée par le découpage syllabique, de telle sorte que le premier segment (la première consonne) marque la fin d'une syllabe et le second segment (la seconde consonne), le début de la syllabe suivante ${ }^{30}$. Cela implique que la seconde consonne soit suivie d'une voyelle, dans une structure de type-CCV. En l'absence de voyelle subséquente, autrement dit, pas de gémination possible. Sans recourir à la notion de syllabe, les anciens savants iraniens l'avaient bien compris. Ils expliquent le phénomène de la façon suivante : « les lettres géminées sont composées de deux lettres : la première, quiescente (sāken), et la seconde, vocalisée (motaharrek). » ${ }^{31}$

Sur les conséquences, il n'y a aucun doute possible : prononcés isolément ou devant consonne, des mots comme par(r) (plume), zar(r) (or), haq(q) (droit, vérité), šak(k) (doute, soupçon), haț(t) (ligne, écriture), teb(b) (médecine), mohem(m) (important), fan(n) (art,

\footnotetext{
25. ȘĀDEQĪ 1392/2013, p. 24.

26. Ṭūsī 1370/1992, p. 179.

27. « Tašdīd-e pāyānī ». ṢĀDEQĪ 1392/2013, p. 30-33 ; VAب̣īDIYĀN KĀMYĀR 1371/1992, p. 24-25.

28. LAZARD 1957, p. 24.

29. VAḤ̂̄DIYĀN KĀMYĀR 1371/1992, p. 24-25.

30. Voir aussi TROUBETZKoY 1949, p. 184, cité ci-dessus.

31. Ṭūsī 1370/1992, p. 179.
} 
technique), zan(n) (supposition, opinion), rob(b) (suc, extrait), sen(n) (âge), qad(d) (stature, taille), sad(d) (digue, barrage) et autres semblables n'ont pas de consonne géminée. On évoquera, à la rigueur, une géminée «sous-jacente ». De fait, si l'on sait que ces mots contiennent un tašdìd étymologique, c'est qu'il arrive que la géminée se réalise, mais uniquement sous certaines conditions : la gémination finale de mot se réalise si et seulement si celui-ci est suivi d'une voyelle ${ }^{32}$. On parlera à leur sujet de variantes combinatoires, en distribution complémentaire. T. Vahīidiyān Kāmyār développe l'exemple du mot haq(q). On a bien une géminée dans le dérivé haqqāniyat (justice, légitimité), dans les expressions haqq-o hoqūq (les droits) et haqq-e man (mon droit), ou dans les phrases haqqī nadārad (il n'a pas le droit), haqq-eš in-ast (c'est son droit). En revanche, la consonne n'est pas redoublée dans haq tout seul, ni dans l'expression haq bā to-st (tu as raison) ${ }^{33}$. La règle est purement phonétique, et s'applique indifféremment selon que la voyelle subséquente est interne au mot (comme dans les dérivés) ou qu'elle appartient à un suffixe, voire au mot suivant ${ }^{34}$.

Plus généralement, le tašdìd « final » soulève la question des conditions de réalisation des géminées, et de leur environnement phonétique. C'est pourquoi on le désigne ici, après les linguistes iraniens, du nom de tašdìd « contextuel » ${ }^{35}$. Or, il semblerait qu'outre le tašdìd final, le persan dispose encore du tašdìd contextuel dans des réalisations d'un autre type. Sur la base des descriptions collectées auprès des auteurs classiques ${ }^{36}$, Șādeqī aboutit ainsi au recensement suivant. En persan, hors gémination « lexicale », le tašdìd peut apparaître :

- à la suite des mots : to (tu, toi), do (deux), se (trois»), na (non), ke (qui, que), baste (fermé), raste (délivré), lāle (tulipe) et borde (porté, transporté), sur la consonne initiale du mot suivant. Exemple : do mmāh (deux mois) ;

- sur la consonne initiale du mot suivant la conjonction de coordination, lorsqu'elle est prononcée -o. Exemple : man-o tto (moi et toi) ;

- sur la consonne initiale du nom annexé, après edāfe (particule d'annexion, réalisée -e ou -ye). Exemple : nām-e hhhodā (le nom de Dieu);

- sur la consonne initiale du verbe à l'impératif précédé du préverbe be-, ou ma- à l'impératif négatif. Exemple : bemmān (reste!) ; makkan (n'arrache pas !) ;

- sur le $y \bar{a}^{j}(-\bar{l})$ précédant l'edāfe. Exemple : bimārīy-e del (langueur du cœur) ${ }^{37}$.

Chacune de ces occurrences est bien attestée. Cette liste soulève toutefois pour le linguiste un certain nombre de problèmes. Le premier, c'est qu'aucun des exemples qui lui

32. Selon A.-A. Șādeqī, les mots à tašdīd final sont pour la plupart d'origine arabe et relèvent de racines dites bilitères. ȘĀDEQİ 1392/2013, p. 33.

33. VAḤIDIYĀN KĀMYĀR 1371/1992, p. 24.

34. A.-A. Șādeqī, en partisan résolu du traitement "persan » des géminées, remarque à ce propos qu'après simplification, nombre de mots arabes à tašdìd final ont définitivement perdu leur géminée, même devant

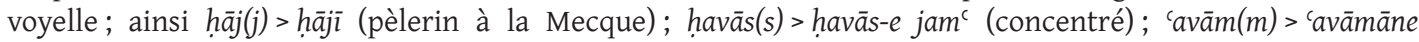
(populaire) ; fan $(n)>$ fan-e $\bar{u}$ (son art, son métier); kaf(f) > kaf-e dast (la paume de la main). ȘĀDEQİ 1392/2013, p. 33-35.

35. « Tašdīd-e bāftī ». ȘĀDEQĪ 1392/2013, p. 38 et passim ; VAḤĪDIYĀN KĀMYĀR 1371/1992, p. 25.

36. ȘĀDEQĪ 1392/2013, p. 4-5.

37. ȘĀDEQİ 1392/2013, p. 6. 
sont associés ne correspond à la pratique standard du persan moderne. Ils correspondent à des états de langue ancien, dialectal ou poétique dont le rattachement au persan courant ne va pas sans difficultés. Le second problème concerne l'interprétation des sources. Les descripteurs classiques, poéticiens ou lexicographes, qui ont livré les contours de ce tašdid contextuel, prenaient pour support quasi exclusif la poésie classique. C'est pourquoi, outre le défi qui consiste à vouloir rendre compte de la pluralité de ces géminées à l'aune de la phonologie moderne, le linguiste se voit confronté à une autre gageure: la nécessité de déterminer si ces manifestations de la gémination sont propres à la poésie, ou si elles se retrouvent également en prose, et dans la langue parlée.

\section{Le tašdīd des poètes: vérité phonétique ou licence poétique?}

Parmi les caractères de la gémination les plus nettement identifiés par N. Troubetzkoy, il en est un dont les conséquences prosodiques sont bien connues. Les consonnes géminées, on l'a vu, sont traversées par le découpage syllabique, de telle sorte qu'elles se répartissent sur deux syllabes successives :-C/CV. On comprend donc que le redoublement consonantique joue un rôle crucial en poésie. Il influe aussi bien sur le nombre de syllabes dans la poésie syllabique que sur le décompte des mores dans la poésie quantitative, ou sur la répartition des accents dans la poésie accentuelle. La poésie persane traditionnelle se fonde sur la métrique quantitative arabe, appelée 'arū ${ }^{38}$. Pour aménager leurs créations à cette métrique exogène, les poètes persans ont, de tout temps, rivalisé d'ingéniosité et recouru à toutes les ressources de leur langue. À ce titre, on s'en doute, les possibilités qu'offrait la gémination n'étaient pas en reste. Mais de là à conclure que la gémination suit en poésie des règles inconnues de la prose, il y a un pas que les linguistes sont réticents à franchir : se pourrait-il qu'il y ait en propre un tašdìd des poètes?

A.-A. Șādeqī témoigne une conscience aigüe du problème. En vérité, sa contribution à l'étude de la gémination se conçoit tout entière comme une réponse à cette question. Plus radicalement que ses prédécesseurs, le linguiste y répond par la négative :

Nombre de savants, anciens et modernes ont affirmé que l'apparition des tašdìd "contextuels" et "analogiques", voire de certains tašdìd "lexicaux" tombés hors d'usage, était imputable aux licences poétiques; mais, bien que cela soit vrai de quelques rares occurrences, c'est fondamentalement faux (asāsan nā-dorost-ast). ${ }^{39}$

À contre-courant de cette croyance, tout l'effort du linguiste consiste à rapatrier sur le terrain de l'analyse phonétique ces géminations qualifiés commodément, et abusivement selon lui, de « licences poétiques » (darūrat-e šăcerì).

Mieux que de longs discours, un ancien adage arabe résume les hantises de tout linguiste: dans l'usage de la langue, «il est permis au poète ce qui n'est pas permis

38. Voir en particulier BOHAS et PAOLI 1997 ; UTAS 1994.

39. ȘĀDEQİ 1392/2013, p. 38. 
aux autres » (yağūzu li-l-šăcir mā lā yağūzu li-ġayri-hi). Jusqu’à quel point le poète peut-il intervenir sur la langue commune ? Et comment le linguiste peut-il rendre compte de ces audaces ? La question des frontières entre langue commune et langue poétique est un souci constant des descripteurs, tant anciens que modernes. À ce titre, la gémination offre une arène de choix pour l'observation des divergences, tant d'opinion que de méthode, parmi les spécialistes. Les usages du tašdìd en poésie fournissent plusieurs cas litigieux. Nous n'en mentionnons ici que quelques-uns parmi les plus fréquents.

\section{Une dégémination impromptue}

Depuis les premiers vers persans, composés aux alentours du $\mathrm{IX}^{\mathrm{e}}$ siècle, la simplification des géminées "lexicales» est sans doute l'une des libertés les plus courantes que s'accordent les poètes. T. Vahīdiyān Kāmyār remarque ainsi que dans un vers, Nāṣer Hosrow (ca. 1004-1074) emploie le mot arabe 'ām(m)e (public, commun) sans sa géminée (mohaffaf) ${ }^{40}$. A.-A. Șādeqī note que le mot korre (au sens de "poulain, ânon ») apparaît chez Sa'dī (ca.1210-1291) avec son tašdīd, mais chez Amīr Hosrow (1253-1325) sans ${ }^{41}$. Les linguistes fournissent chacun des exemples; ils n'en diffèrent pas moins dans l'interprétation qu'ils en donnent. T. Vahịidiyān Kāmyār fait valoir que les poètes ont, de tout temps, su tirer profit de la double lecture possible du tašdid lexical, employant la variante " allégée » quand bon leur semblait. Plus encore, ils n'ont pas hésité, selon lui, à étendre cette option aux termes qui ne semblaient pas l'autoriser ${ }^{42}$. Pour sa part, A.-A. Șādeqī ne cite que des cas où la simplification des géminées est une variante phonologique connue et admise. C'est notamment le cas du [r] géminé à l'intervocalique, qu'il est toujours, dit-il, loisible de simplifier.

\section{Un tašdīd superfétatoire}

Les divergences d'analyse sont plus tranchées au sujet de l'emploi par les poètes de géminées étymologiquement imprévisibles. Plus rare que la dégémination, l'ajout de géminées produit aussi un effet plus inhabituel dans la langue. T. Vahīdiyān Kāmyār reconnaît ainsi que le tašdid pratiqué par Nāsser Hosrow - encore lui - sur harr (âne) et zebbar (au-dessus) dans un poème ne contrarie pas seulement l'usage courant, mais les principes mêmes de «l'éloquence » (faṣāhat ${ }^{43}$. Le phonéticien range tous ces usages sous la bannière des « licences poétiques » (darūrat-e še $e^{r} i \overline{)}$ ), et en rend principalement responsables les poètes anciens. À l'en croire, les poètes tardifs s'en tiennent le plus souvent au jeu

\footnotetext{
40. VAḤIDIYĀN KĀMYĀR 1371/1992, p. 27.

41. Ș̣ĀDEQĪ 1392/2013, p. 26.

42. VAḤīDIYĀN KĀMYĀR 1371/1992, p. 27.

43. Ibid. Notons que Šams-e Qeys jugeait déjà sévèrement l'ajout de géminées dans les mots où elles n'ont rien à faire - pratique qu'il compte au nombre des « ajouts hideux ». Dans les mots d'origine arabe, en particulier, le tašdìd excédentaire trahit l'ignorance du poète eu égard à la racine du mot (il donne pour exemple un vers de Hāāānī qui gémine le [m] dans le prénom 'Ommar). Šams-e Qeys 1373/1995, p. 267; trad. DIEBLER 1997, p. 210.
} 
des variantes permises par les tašdìd « libres », tandis que les poètes des premiers siècles ajoutaient ou supprimaient des géminées « sans égard aux règles de la langue (bar helāf-e $m e^{c} y a \bar{r}-e$ zabān) ${ }^{44}$. À ces affirmations, A.-A. Șādeqī répond par l'extension du domaine du tašdìd historique. Il prend à témoin tantôt l'étymologie, tantôt les variantes dialectales, ou la durée articulatoire des consonnes fricatives en position intervocalique pour justifier que madres(s)e (école), mī-res(s)e (il arrive), mās(s)e (sable), $k a m(m) \bar{l}$ (un peu), mož(ž)e (cil) puissent être réalisés avec tašdìd ${ }^{45}$. Si bien qu'en définitive, il fournit de ce type d'occurrence une explication inverse de celle de T. Vahīdiyān Kāmyār. Pour le linguiste, la présence du tašdìd est presque toujours justifiable historiquement; seulement, sa réalisation demeure facultative : le mot peut aussi bien se prononcer sans (mohaffaf).

Le tašdīd du mot de la fin

Nous avons vu que le tašdìd final de mot soulevait la question de son environnement phonétique : il ne se réalise habituellement que devant voyelle. En poésie, les mots à tašdid final suscitent deux types d'interrogations : comment les lire s'ils apparaissent à la rime? Et peuvent-ils se rencontrer en l'absence de voyelle subséquente?

Pour T. Vahịidiyān Kāmyār, la première question se laisse aisément résoudre. Il s'agit selon lui d'un faux problème. Les rimes, rappelle-t-il, sont un sûr étalon pour la prononciation; quand un mot à tašdìd final se retrouve à la rime, il se prononce sans gémination, comme on doit s'y attendre devant sokūn ${ }^{46}$. Ainsi, Nāṣer Hosrow fait rimer zan(n) (supposition, opinion) et fan(n) (art, technique) avec man (moi) et bahman (nom du onzième mois de l'année iranienne); Hāaānī (ca.1127-1199) fait rimer far(r) (faste, splendeur) avec ahdar (vert); Ḥăfeẓ (ca.1315-1390) fait rimer šak(k) (doute) avec namak (sel) et malak (ange). Autrement dit, lorsqu'ils les emploient à la rime, c'est une rime sans géminée que construisent les poètes. Contrairement aux modifications examinées précédemment, il ne s'agit donc pas d'une simplification des géminées, et encore moins d'une licence poétique, mais bien de la règle générale de distribution des géminées finales de mot, réalisées seulement devant voyelle.

Sur ce chapitre, A.-A. Șādeqī réfute explicitement son prédécesseur. Pour le linguiste, la seule règle qui interdise la réalisation du tašdỉd final à la rime n'est pas d'ordre phonétique, mais métrique ${ }^{47}$. En effet, explique-t-il, il arrive, chez les poètes persans les plus anciens, que le tašdid final de mot soit réalisé dans le corps du vers, sans voyelle subséquente. Ces occurrences suffisent à invalider l'incontestabilité de la règle de distribution complémentaire du tašdìd final (présence ou absence de voyelle subséquente), que T. Vahīdiyān Kāmyār présentait comme inviolable. Toutefois, A.-A. Șādeqī a soin de préciser que l'apparition d'une géminée devant consonne est toujours compensée, phonétiquement,

\footnotetext{
44. VAḤ̄DIYĀN KĀMYĀR 1371/1992, p. 27.

45. ȘĀDEQĪ 1392/2013, p. 26-29.

46. VAḤīDIYĀN KĀMYĀR 1371/1992, p. 25.

47. ȘĀDEQĪ 1392/2013, p. 30-33.
} 
par l'apparition d'un schwa épenthétique $\left(-\mathrm{CC}^{\mathrm{v}}\right)^{48}$. Ce phénomène, requis par le mètre, se traduit par un allongement de la durée articulatoire et correspond à l'ajout d'une syllabe brève à la syllabe longue formée par la syllabe fermée précédente. Le résultat n'est autre que ce que les métriciens iraniens ont appelé «syllabe surlongue » (hejā’-e kešìde) : (- U). Or, c'est une règle de base de la métrique arabo-persane que la dernière syllabe du vers se définit comme anceps, c'est-à-dire, indifférente à la quantité. C'est pourquoi, il n'y a pas de sens à parler de syllabe surlongue à la rime. Ainsi, pour A.-A. Șādeqī, on peut trouver des consonnes géminées en l'absence de voyelle subséquente : elles se prononcent à l'aide d'un schwa; en revanche, on ne saurait les trouver à la rime, c'est-à-dire, dans la dernière syllabe du vers. Du point de vue de A.-A. Șādeqī, tous les usages du tašdīd doivent trouver une explication phonétique. Alors, si « licence poétique » il y a, c'est dans le choix que font parfois les poètes de recourir à des prononciations rares ou archaïques.

Une chose est sûre : dans toutes les occurrences de géminées anomales attestées en poésie, c'est le mètre du poème qui permet de déterminer la place et la nature exacte du tašdìd, ou de la dégémination inattendue. De semblables irrégularités se constatent aussi bien dans la poésie arabe. Simplification ponctuelle des géminées, ou à l'inverse, ajout d'une géminée où elle ne serait pas tolérée en prose, notamment en finale de mot: W. Wright lui-même en recense quelques cas dans sa grammaire ${ }^{49}$. Parmi les manifestations irrégulières du tašdìd, il reste cependant un cas que nous n'avons pas encore évoqué. Cette catégorie, qui avait retenu l'attention des anciens descripteurs et spécialistes de la poésie persane, correspond à ce que l'on pourrait appeler, par contraste avec le précédent, le tašdìd « initial de mot ». C'est aussi la figure exemplaire de la rubrique annoncée en introduction sous le nom de tašdìd « métrique ».

\section{Le tašdīd métrique}

Pour bien comprendre ce qui se joue avec le tašdìd en poésie, il convient de rappeler cette évidence : dans un poème, la métrique impose de lire chaque hémistiche comme un seul long mot phonétique. En conséquence, les syllabes se trouvent redistribuées dans la chaîne parlée, et les frontières de mots, abolies. Nașīr al-Dīn Ṭūsī ne disait pas autre chose lorsqu'il précisait, dans son traité de métrique : «quand nous parlons de lettres vocalisées et quiescentes, notre propos vise les lettres prononcées (malfūz), non les lettres écrites $\left(\right.$ maktūb) ${ }^{50}$. C'est précisément là qu'intervient le tašdìd contextuel : il se produit le plus souvent à la frontière des mots, ou comme dirait N. Troubetzkoy, «à la jointure des morphèmes ${ }^{51}$, pour assouplir la langue aux proportions exigées par le mètre. En dehors

\footnotetext{
48. ȘĀDEQĪ 1392/2013, p. 30.

49. WRIGHT 1951, IV Prosodie, « licences poétiques », §237-238, p.377-378. Voir aussi BoHAS et PAOLI 1997 ; RETSÖ 1994.

50. Țūsī 1370/1992, p. 179.

51. TROUBETZKOY 1949, p. 185.
} 
du tašdìd final, en effet, les descripteurs anciens recensent encore plusieurs types de tašdìd contextuels, si bien que cette catégorie excède largement, en persan, celle du tašdïd lexical.

Le tašdīd initial de mot

Le tašdīd initial de mot ne se rencontre qu'en poésie. Un exemple suffira à l'illustrer. En effet, A.-A. Șādeqī cite le vers suivant du poète Manūčehrī (m. ca. 1040) ${ }^{52}$, qui en contient trois occurrences :

Man-o tto ġgāâfelìm-o mmāh-o horšìd // Bar-īn gardūn-e gardān nì-st ġāfel ${ }^{53}$

Ni toi ni moi nous n'y pensions, mais la lune et le soleil // Ne sont pas inattentifs à cette sphère qui tourne sans cesse ${ }^{54}$.

Deux des cinq types de tašdīd contextuels recensés par les descripteurs anciens ${ }^{55}$ se réalisent dans le premier hémistiche. Il s'agit d'une part du tašdìd touchant la consonne suivant un mot à finale vocalique (ici : to ġgáafelìm); d'autre part, du tašdìd portant sur la consonne suivant la conjonction de coordination [-o] (deux exemples ici : man-o tto ; $\dot{g} a \bar{f}$ elìm-o $m m \bar{a} h$ ).

Par contraste, une lecture non métrique, en persan courant, de ce vers, donnerait :

Man-o to ġäfelìm-o māh-o horš̌̀d bar-īn gardūn-e gardān nī-st gàafel.

Or, le poème se fonde sur le mètre hazaj-e mosaddas-e maḥ̂ūuf (ou maqșūr), qui correspond dans la métrique traditionnelle au modèle mafāicilun mafäcìlun facūlun, soit à la scansion syllabique suivante : $\mathrm{U}_{---} / \mathrm{U}---/ \mathrm{U}--(\mathrm{U})$. On le comprend : l'ajout de consonnes géminées a pour fonction de produire des syllabes longues, de façon à conformer l'hémistiche au mètre du poème :

\begin{tabular}{|c|c|c|c|c|c|c|c|c|c|c|}
\hline$M a$ & not & to $\dot{g}$ & $\dot{g} \bar{a}$ & fe & lì & mom & $m \bar{a}$ & ho & hom & šìd \\
\hline$m a$ & $f \bar{a}$ & $c_{\bar{l}}$ & lun & $m a$ & $f \bar{a}$ & ${ }^{\prime} \bar{\imath}$ & lun & $f a$ & ${ }^{\prime} \bar{u}$ & lun \\
\hline$U$ & - & - & - & $U$ & - & - & - & $U$ & - & (U) \\
\hline
\end{tabular}

De fait, pour transformer en syllabe longue une syllabe phonétiquement brève [consonne + voyelle brève], il n'y a que deux solutions : l'une consiste à allonger le noyau vocalique de la syllabe, pour avoir une structure [consonne + voyelle longue]; l'autre consiste à ajouter une consonne après la voyelle brève, pour obtenir une syllabe fermée de type [consonne + voyelle brève + consonne].

52. Abū’n-Najm Ahmad b. Qowṣ Manūčehrī Damḡānī (m. ca. 1040). Né à Damġān vers la fin du X ${ }^{\mathrm{e}}$ siècle, il vécut à la cour du Ziyaride Manūčehr b. Qābūs (regn. 1012-31) avant de servir le sultan Mas'ū de Ġazna (regn. 1030-40).

53. Vers cité par Ṣādeqī 1392/2013, p. 9. Nous signalons la position des géminées par des caractères gras.

54. Manūčehrī 1886, qașìda 29, v. 7, p. 74 ; trad. KAZIMIRSKI, p. 207.

55. ȘĀDEQĪ 1392/2013, p. 6. 
L'allongement des voyelles brèves fournit la justification moderne des variations de quantités syllabiques dans le vers persan. Ainsi, on admet d'ordinaire qu'en persan, toute voyelle brève en syllabe ouverte (en position de suffixe ou en finale de mot) est susceptible d'allongement. C'est ce qui permet d'expliquer que, dans certains vers, la syllabe longue exigée par le mètre se réalise par une voyelle phonétiquement brève. Mais pour avancer une telle explication, il va de soi qu'il faut disposer de la notion de syllabe, et des principes de la phonologie moderne.

La métrique arabo-persane traditionnelle se fondait quant à elle sur la combinaison des lettres écrites, vocalisées et quiescentes. Elle ne connaissait pas les syllabes, longues ou brèves, mais se fondait sur des éléments métriques de base composés de consonnes vocalisées et quiescentes, appelés sabab et watid. Le « sabab léger » (sabab-e hafiff) désigne le composé $[\mathrm{CV}+\mathrm{C}]$, qui correspond à une syllabe longue (-).

\begin{tabular}{ll}
\hline Syllabe brève $\mathrm{CV}=\mathrm{U}$ & Syllabe longue - \\
\hline Allongement vocalique & $\mathrm{CV}=-$ \\
Ajout de consonne & $\mathrm{CvC}=-(=$ sabab-e hafif $)$ \\
Gémination & $\mathrm{CvC}-\mathrm{C}(\mathrm{v})$ \\
\hline
\end{tabular}

En géminant la consonne suivant la voyelle brève, on obtient un « sababléger » (pour les modernes : une syllabe fermée), autrement dit, la syllabe longue que l'on recherchait. Ainsi, les métriciens classiques s'en remettaient à la gémination pour expliquer les variations quantitatives dans la phonologie du persan. Voyons comment ils s'en acquittaient en leurs temps.

\section{Vieux métriciens...}

Pour Nașīr al-Dīn Ṭ̂̄sī, « le tašdìd se rencontre en persan dans deux circonstances » ${ }^{56}$, dont le tašdìd lexical ne constitue qu'un cas de figure. Car la gémination, précise-t-il, peut aussi se produire « entre deux mots »,

sur la première lettre du mot coordonné ( $m a^{c} t$ țuf), du nom annexé (mudâfun ilay-hi) ou du mot précédé du $b \bar{a}^{\prime}$ de l'ordre (b $\left.\bar{a}^{\prime}-e a m r\right)$ ou du mìm de la défense (mim-e nahy), comme dans les mots bekkan (arrache!) et makkan (n'arrache pas!), ou bien encore, sur une lettre qui n'apparaît pas dans la prononciation, tels le vāv de dow (deux) et de tow (toi), le ha $\bar{a}$ de seh (trois), de nah (non), de čeh (car, quel), de keh (que, qui), de lāleh (tulipe) et de bordeh (emporté). En dehors de pareilles circonstances, le tašdìd est laid (qabih), et il n'est obligatoire dans aucune de ces occurrences bien qu'il soit courant $(\operatorname{rav} \bar{a}) .{ }^{57}$

56. Ṭ̂̄sī 1370/1992, p. 179. Voir aussi les commentaires de Morādābādī dans Ṭūūì 1389/2011, p. 189-190.

57. Ṭūsī 1370/1992, p. 179. Les caractères gras soulignent la position du tašdìd. Notons que la « laideur » associée ici aux autres cas de gémination renvoie moins à un jugement esthétique proprement dit qu'à un jugement d'acceptabilité fondé sur des critères d'usage. Sur cette question, voir PAOLI 2008, p. 255-281. En l'occurrence, les séquences phoniques sont appréciées en termes de «lourdeur » et de "légèreté » relatives à l'effort articulatoire, selon des critères établis par les grammairiens de l'arabe classique. Sur ce point, voir BoHAS 1981 et Kouloughli 1986. 
À première vue, une telle recension peut paraitre déconcertante. Les exemples proposés semblent ne refléter aucune réalité phonétique. En persan, en effet, la lettre-consonne ( $v \bar{a} v$ ou ha' « muet ») qui clôt dans la graphie les mots à finale vocalique ${ }^{58}$ est un simple support de voyelle qui n'est jamais prononcé ; autrement dit, une consonne-fantôme. De plus, les occurrences citées cumulent les complications rencontrées dans les géminées « de poète » : elles associent l'impression d'arbitraire produite par les géminées non étymologiques et l'artifice du tašdìd final de mot. En d'autres termes, elles ne semblent pas "naturelles" dans la langue. Enfin, que peut bien être ce tašdìd qui intervient, selon l'auteur, «sur une lettre qui n'apparait pas dans la prononciation (harfi [...] ke dar lafz nayāyad) »?

Son contemporain Šams-e Qeys fournit un complément de réponse. Ses remarques sur la gémination se rencontrent dans son traité sous la rubrique des « vices de rimes » ${ }^{59}$. Il convient de lire l'ensemble du passage pour comprendre l'argument. Šams-e Qeys énumère alors «les trois cas où les consonnes sont redoublées». Outre le cas du redoublement de " la consonne rā") ", qui correspond à un tašdìd lexical, un autre, dit-il, « est le cas des mots dont la finale est une consonne non prononcée, comme do(w) (deux), to(w) (tu), ne(y) (ne... pas), ke(y) (qui), baste(h) (lié), raste(h) (échappé) et mots semblables, où la vocalisation de ce qui précède relie le $v \bar{a} v$, le $y \bar{a}$ ' et le $h \bar{a}$ ' à ce qui les suit, si bien qu' il nait un redoublement qui remplace ces consonnes, comme dans : « Do mmāh šod ey dūst ke to hhejr gozìdī, Deux mois sont passés, mon amie, depuis que tu as élu de partir.»

Et une autre encore dans la coordination ou dans la détermination. C'est ainsi que les Anciens ont dit : « Man-o tto-īm negārā ke 'ešq-o hūbīi-rā // Ze na't-e Leylì-o Majnūn borūn barīm hamī, Nous sommes, toi et moi, ma beauté, tels que nous portons // L'amour et la beauté au-delà du portrait de Leylī et Majnūn » où, puisque ce qui précède le vāv de coordination n'est pas prononcé sous sa forme pure, on affecte une vocalisation o à ce qui précède en le liant à ce qui suit. Si l'on met un redoublement en cette circonstance, il y a également corruption de contraction.

Et dans la détermination, comme dans : «Dar zelāl-e jjäh-e to ārāyeš-ī dārad bašar // Dar jamāl-e "adl-e to āsāyeš-i dārad jahān, Dans l'ombre de ta grandeur, l'humanité trouve sa parure, // Dans la beauté de ta justice, l'univers trouve son repos " pour la raison que la forme de la détermination ne s'exprime correctement en persan que par la vocalisation de la fin du mot déterminé, comme dans yār-e man (mon compagnon) et dans kār-e dūst (le travail de l'ami). «Or, tout mot dont la finale est vocalisée, dans cette langue, exige une consonne muette, que cette dernière entre ou non dans la prononciation, ainsi que nous l'avons montré dans le cas de to(v), do(v), na(h), se(h) (trois), gofte(h) (dit) et raste(h); pour cette raison, il s'ensuit que, entre le mot déterminé et le mot déterminant, la corruption d'une contraction se fait jour et, à cet égard, le redoublement de l'initiale du

58. $V \bar{a} v(g)$ la fin de do et de to ; hä'-e sakt (a) à la fin de se, na, če, ke, lāle et borde.

59. Šams-e Qeys 1373/1995, p. 267-268; trad. DIEBLER 1997, p. 211-212. 
mot déterminant n'est pas laid. Et, hormis ces trois circonstances, le redoublement des consonnes n'est pas agréé » ${ }^{60}$.

Ici, le lexicographe est plus explicite que le philosophe. Nașīr al-Dīn évoquait, de manière sibylline, la gémination d' « une lettre qui n'apparaît pas dans la prononciation ». Šams-e Qeys précise qu'en réalité, « il naît un redoublement qui remplace ces consonnes ». Autrement dit, il y a déport du tašdìd de la « consonne non prononcée » (harf-e ġeyr-e malfūză) sur la consonne initiale du mot subséquent. Dans l'exemple de vers cité, en effet, on a bien : do mmāh šod et non pas *dow māh ; to hhejr et non pas * tow hejr.

Dans toutes ces occurrences, c'est la présence de la voyelle - qu'elle soit enclitique, comme dans la conjonction de coordination ou dans la particule d'annexion, ou finale de mot - qui, en « liant » «ce qui précède (...) à ce qui suit », raccorde les uns aux autres les phonèmes successifs. C'est cette voyelle, en d'autres termes, qui permet au poète de traiter la première consonne du mot suivant comme si elle se trouvait à l'intérieur du mot, et non à l'initiale, condition théorique indispensable à la gémination. Ainsi, « le redoublement de l'initiale du mot » dont parle Šams-e Qeys n'en est pas un, puisque les frontières de mots sont inopérantes dans la chaîne phonique du vers. Ou plutôt, le tašdìd « initial de mot » ne peut être qu'un tašdìd de poète, c'est-à-dire, une variable d'ajustement métrique.

\section{... et jeunes linguistes}

Pour rendre compte de la labilité des géminées en poésie, les auteurs du XIII ${ }^{\mathrm{e}}$ siècle avaient su développer des arguments sophistiqués. Les considérations qui précèdent ne témoignent pas seulement de leur parfaite intelligence des phénomènes phonétiques, mais également du souci de maintenir une distinction nette entre l'analyse du discours ordinaire et celle des conventions propres à la langue poétique. En effet, Šams-e Qeys comme Nașīr al-Dīn ont soin de préciser que ce tašdìd indifférent aux frontières de mots ne se comprend qu'en poésie. Ils n'en tirent aucune conclusion relative à la prose ou à la langue parlée. Cette prudence méthodologique est sans doute ce qui les distingue des linguistes de notre temps.

Pour Șādeqī, en effet, tout est question de point de vue. Loin d'ignorer la perspective des descripteurs médiévaux, il cite les analyses de Šams-e Qeys et de Nașīr al-Dīn dans leur intégralité, incluant au passage quelques-uns de leurs successeurs ${ }^{61}$. Ce matériau fournit même la base de sa réflexion. Seulement, son ambition à lui est de dresser un panorama exhaustif de la gémination en persan. Que ce soit en prose ou en poésie, il cherche à rendre compte de manière unifiée de la diversité des phénomènes de gémination afin de réduire ces derniers, dans la mesure du possible, à quelques règles générales ${ }^{62}$. C'est donc tout naturellement vers la phonétique historique qu'il se tourne pour rendre raison des

60. Šams-e Qeys 1373/1995, p. 268 ; trad. DIEBLER 1997, p. 211-212.

61. 'Abd al-Rašīd Tatavī, auteur du Farhang-e Rašìdī, Hamze b. Yūsof Sahmī, auteur du Tārīh-e Jorjān, et Najm al-Ġanī Hān. Pour l'essentiel, les citations puisées chez ces auteurs se contentent de relayer l'une ou l'autre catégorie de gémination définie par Ṭūsī ou par Šams-e Qeys. Voir ȘĀDEQİ 1392/2013, p. 4-5.

62. ȘĀDEQĪ 1392/2013, p. 6. 
occurrences les plus variées. Le linguiste pose ainsi que le tašdìd contextuel ne saurait se manifester que dans une position jadis occupée par une voyelle longue historique, que celle-ci ait ou non disparu depuis ${ }^{63}$. De cette manière, tous les cas se laissent aisément expliquer. Les mots to, do, se qui s'achèvent aujourd'hui par une voyelle brève étaient jadis, en moyen perse, dotés d'une voyelle longue $(d \bar{o}, t \bar{o}, s \bar{e})$. La gémination de la consonne subséquente illustrée par Šams-e Qeys (do mmāh šod) se laisse alors comprendre comme un archaïsme, c'est-à-dire, comme une réaction phonétique tardive à l'abrègement consonantique ${ }^{64}$.Il en va de même de la conjonction de coordination [-o], anciennement [ $\left.\bar{u}\right]$, du moyen perse $[u d]{ }^{65}$, et de la particule d'annexion : un [i] long en moyen perse ${ }^{66}$.

Șādeqĩ étend encore ce principe explicatif aux exemples d'impératif (bekkan; makkan) rapportés par Nașir al-Dīn. Prenant à témoin des réalisations dialectales contemporaines, le linguiste émet l'hypothèse, non autrement démontrable, d'une ancienne voyelle longue dans les préverbes [be-] et [ma- ${ }^{67}$. Enfin, lorsqu'il s'interroge sur l'apparition exclusive de ces géminées en poésie ${ }^{68}$, le linguiste en appelle, une fois encore, à l'argument historique: «De nos jours, dans de telles circonstances, nous prononçons la conjonction de coordination et la particule d'annexion [comme des voyelles] allongées ; mais autrefois, ces dernières étaient prononcées brèves, et la consonne subséquente était géminée » ${ }^{69}$. Qu'ils lui accordent le statut de "licence poétique », à l'instar de T. Vahịidiyān Kāmyār, ou qu'ils s'y refusent, les linguistes d'aujourd'hui ne voient ainsi dans le tašdìd contextuel qu'un archaïsme de poètes, fidèlement enregistré par les anciens descripteurs de la langue.

Au regard de la phonétique historique, le raisonnement est sans doute irréprochable et la conclusion, jusqu'à preuve du contraire, doit être tenue pour vraie. Force est d'admettre que le tašdìd contextuel, lorsqu'il se réalise, est le lointain témoin d'un ancien événement phonétique. La langue de la poésie, c'est bien connu, est conservatrice. Elle garde la trace de sons dont la langue courante a perdu tout souvenir. Pour les linguistes, l'abrègement historique des voyelles longues, même antérieur aux plus anciens poèmes persans, serait ainsi commémoré à travers les siècles par les poètes dans ces géminations différées. Pourtant, dans cette version de l'histoire de la gémination, un fait reste passé sous silence. Un fait mineur, sans doute, aux yeux des chercheurs modernes, puisqu'il relève moins de la science que du discours de science - mais non moins remarquable au regard de l'histoire des idées, et de la pensée du persan. Ce fait, c'est l'avènement du tašdīd comme outil descriptif sous la plume des poéticiens classiques, qui l'érigèrent en véritable opérateur d'ajustement entre la métrique et les sons de la langue.

63. ȘĀDEQĪ 1392/2013, p. 7 : « haqīqat inn-ast ke zohūr-e tašdìd dar kolliye-ye in mavāred marbūṭ be bāft-hā-yī-st ke yek moșavvet-e boland-e tārīhī dar ān-hā vojūd dārad, hwwāh in moșavvet-e boland hanūz dar bāft-hā-ye mowred-e nazar mowjūd bāšad, ḩwāh az beyn rafte bāšad ».

64. ȘĀDEQİ 1392/2013, p. 7-8.

65. Ibid., p. 8-9.

66. Ibid., p. 9.

67. Ibid., p. 9-10.

68. Ibid., p. 6.

69. Ibid., p. 9. 


\section{Conclusion}

Avec le tašdìd " métrique ", les anciens descripteurs ne se munissaient pas seulement d'un expédient utile pour effacer les discords entre la mesure du vers et la mesure de la langue; ils se dotaient d'un instrument théorique puissant, qui permettait de traiter la langue poétique pour elle-même, séparément des vicissitudes de la langue commune. Ce tašdìd ne justifiait aucun phénomène en langue; il expliquait l'apparition en poésie de géminées non lexicales, et qui plus est facultatives, dans des positions d'où elles seraient aussi bien absentes si elles n'étaient requises par le mètre. À l'ouverture de son traité, Šams-e Qeys distinguait les plans d'emblée : «Sache que la métrique ('arūḍ) est la balance (mizān) du discours en vers, de la même manière que la grammaire est la balance du discours en prose ${ }^{70}$, prévient-il son lecteur.

De fait, si l'on s'en tient aux définitions des anciens, on serait bien en peine d'identifier les phonèmes correspondants dans la chaîne des sons. La gémination d'« une lettre qui n'apparaît pas dans la prononciation ${ }^{71}$, ou qui « remplace » la « consonne non prononcée ${ }^{72}$ produit-elle vraiment deux consonnes ? Ou se réalise-t-elle dans l'allongement, à peine perceptible, de la voyelle précédente, comme en produisent aujourd'hui les Iraniens dans la déclamation, pour souligner le mètre poétique ? On est en droit de se le demander. Il revient aux historiens de la langue de proposer des reconstitutions; les poéticiens classiques avaient déterminé de ne pas trancher. À l'image du petit signe suscrit dont la légende attribue l'invention à al-Hुalìl, il se peut que le tašdìd des métriciens iraniens ait eu pour seule fonction de compter, non les consonnes effectivement prononcées, mais seulement le rythme, et les mores en poésie. Si c'est le cas, alors, les consonnes de la poésie, réelles ou supposées, lorsqu'elles sont marquées du tašdìd, pourraient toujours valoir pour un peu plus, ou un peu moins que deux.

70. Šams-e Qeys 1373/1995, p. 38 ; trad. DIEBLER 1997, p. 15.

71. Țūsī 1370/1992, p. 179.

72. Šams-e Qeys 1373/1995, p. 268 ; trad. DIEBLER 1997, p. 211. 


\section{Bibliographie}

\section{Sources}

Ibn al-Nadīm, Al-fihrist, éd. I. RAMAIĀN, Beyrouth, Dār al-ma'rifa, 1994.

Manūčehrī Dāmġānī, Menoutchehri, poète persan du $11^{e}$ siècle, éd. et trad. A. DE BIBERSTEIN KAZIMIRSKI, Paris, Klincksieck, 1986.

Šams al-Dīn Moḥammad b. Qeys al-Rāzī, Al-mocjam fī ma‘āyīr aš‘ār al-'ajam, éd. S ŠAMīsĀ, Téhéran, Entešāāāt-e Ferdows, 1373/1995.

Ṭūsī, Nașīr al-Dīn, Me yyār al-ašcār dar 'elm-e 'arū dar ātāar-e ḩَāje nașir al-dīn țūsī, Téhéran, Sāzmān-e Čāp va-Entešārāt-e Vezārat-e Farhang va-Eršād-e Eslāmī, 1370/1992.

Ṭūsī, Nașīr al-Dīn, Me'yāar al-ašcār (dar 'elm-e 'arūd va-qavāfi), dans Mohammad FEŠĀRAKİ (éd.), Me'yār

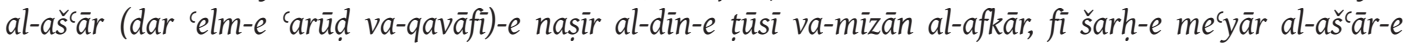
mohammad sa'd allāh moftì morādābādī, Téhéran, Mīrāt-e Maktūb, 1389/2011.

Études

AlfozAN Abdulrahman Ibrahim 1989, Assimilation in Classical Arabic: A Phonological Study, thèse de doctorat, Glasgow, Université de Glasgow.

ADĪB-E SolṬ̂̄Nī Mīr Šams al-Dīn 1354/1975, Dar-āmadī bar čegūnegīe šìve-ye hatțte fārsī, Téhéran, Amīr Kabīr.

BOHAs Georges 1981, «Quelques aspects de l'argumentation et de l'explication chez les grammairiens arabes », Arabica 28/2-3, p. 204-221.

Bohas Georges, Guillaume Jean-Patrick et Kouloughli Djamel Eddine 1990, The Arabic Linguistic Tradition, Londres/New York, Routledge.

BoHAS Georges et PAOLI Bruno 1997, Aspects formels de la poésie arabe, Toulouse, AMAM.

DÉROCHE François 1992, The Abbasid Tradition: Qur'ans of the 8th to the 10th Centuries A.D., London/Oxford, The Nour Foundation, Azimuth Editions and Oxford University Press.

DIEBLER Stéphane 1997, Šams al-Din Mohammad b. Qeys al-Rāzī, Le Livre de la somme, sur les étalons des poésies des Persans, trad. Stéphane DIEBLER, mémoire de D.E.A. d'Études Iraniennes, Paris, Université Sorbonne nouvelle - Paris III, 2 vol.

DutTon Yasin 1999, «Red Dots, Green Dots, Yellow Dots and Blue: Some Reflections on the Vocalisation of Early Qur'anic Manuscripts - Part I », Journal of Qur'anic Studies 1/1, p. 115-140.

FLEISCH Henri 1961, Traité de philologie arabe, vol.1: Préliminaires, phonétique, morphologie, Beyrouth, Imprimerie Catholique.

FLEISCH Henri 1971, «Idghām », Encyclopédie de l'Islam, 2e éd., Leyde/Paris, E.J. Brill et G.-P. Maisonneuve, vol. III, p. 1013-1014.

Kouloughli Djamel Eddine 1982, «Sur la phonographématique de l'arabe », Analyses théorie 1, p. 79-151.

KouloughLi Djamel Eddine 1986, "Sur la structure interne des syllabes "lourdes" en arabe classique », Revue québécoise de linguistique 16/1, p. 129-154.

LANDAU Justine 2013, De rythme \& de raison. Lecture croisée de deux traités de poétique persans du XIII siècle, Paris, Presses de la Sorbonne Nouvelle et Institut Français de Recherche en Iran. 
LAZARD Gilbert 1957, Grammaire du persan contemporain, Paris, Klincksieck.

PAOLI Bruno 2008, De la théorie à l'usage. Essai de reconstitution du système de la métrique arabe ancienne, Damas, Institut français du Proche-Orient.

RETSÖ Jan 1994, "The treatment of final syllables in the classical Arabic metres: the linguistic background », in L. JoHANSON et B. UTAS (éd.), Arabic Prosody and its Applications in Muslim Poetry, Istanbul-Stockholm, Swedish Research Institute in Istanbul [Transactions, vol. 5], p. 99-106.

RIEU Charles 1894, Supplement to the Catalogue of the Arabic Manuscripts in the British Museum, London, The British Museum.

ȘĀDEQĪ 'Alī-Ašraf 1975, «L'influence de l'arabe sur le système phonologique du persan", La Linguistique 11/2, p. 145-152.

ȘĀDEQİ 'Alī-Ašraf 1392/2013, « Tašdīd dar zabān-e fārsī », Farhangnevīsī 5-6, p. 3-43.

SOURDEL-THOMINE Janine 1978, « Khatț. [Writing] i. in the Arab World », dans P. BEARMAN et al. (éd.), Encyclopaedia of Islam, Second Edition, Leiden, Brill, vol. IV, p. 1113-1122.

TRoubETZKoy Nicolaï Sergueevitch 1949, Principes de phonologie, trad. J. CANTINEAU, Paris, Klincksieck.

UTAS Bo 1994, « Arabian and Iranian elements in New Persian prosody », in L. JoHANson et B. UTAS (éd.), Arabic Prosody and its Applications in Muslim Poetry, Istanbul/Stockholm, Swedish Research Institute in Istanbul, [Transactions, vol. 5], p. 129-141.

VAḤIDIYĀN KĀMYĀR Taqī 1371/1992, « Barresī-e tašdīd az dīd-e 'elmī va hall-e yek moškel-e emlā’ì », Rašd-e āmūzeš-e zabān va adab-e fārsī 29-30, p. 24-27.

VeRSTEegh Kees 1997, The Arabic Language, Edinburgh, Edinburgh University Press.

WRIGHT William 1951, A Grammar of the Arabic Language, Translated from the German of Caspari and Edited with Numerous Additions and Corrections, $3^{\mathrm{e}}$ éd. revue et corrigée par W.R. SMITH et M.J. DE GOEJE, Cambridge, Cambridge University Press, 2 vol. 